\title{
Structural and biochemical investigation of class I ribonucleotide reductase from the hyperthermophile Aquifex aeolicus
}

Daniel Rehling ${ }^{1 *}$, Emma Rose Scaletti ${ }^{1 *}$, Inna Rozman Grinberg ${ }^{1 *}$, Daniel Lundin ${ }^{1}$, Margareta Sahlin $^{1}$, Anders Hofer ${ }^{2}$, Britt-Marie Sjöberg ${ }^{1}$ and Pål Stenmark ${ }^{1,3}$

${ }^{1}$ Department of Biochemistry and Biophysics, Stockholm University, S-106 91, Stockholm, Sweden.

${ }^{2}$ Department of Biochemistry and Biophysics, Umeå University, SE-907 36 Umeå, Sweden.

${ }^{3}$ Department of Experimental Medical Science, Lund University, Lund 22100 , Sweden.

Correspondence should be addressed to Britt-Marie Sjöberg, email: $\underline{\text { britt- }}$ marie.sjoberg@dbb.su.se* or Pål Stenmark, e-mail: stenmark@dbb.su.se*

* These authors contributed equally to this work 


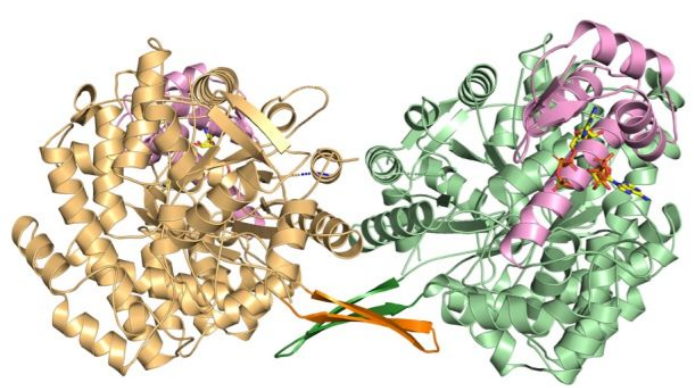

A. aeolicus $\mathrm{R} 1$

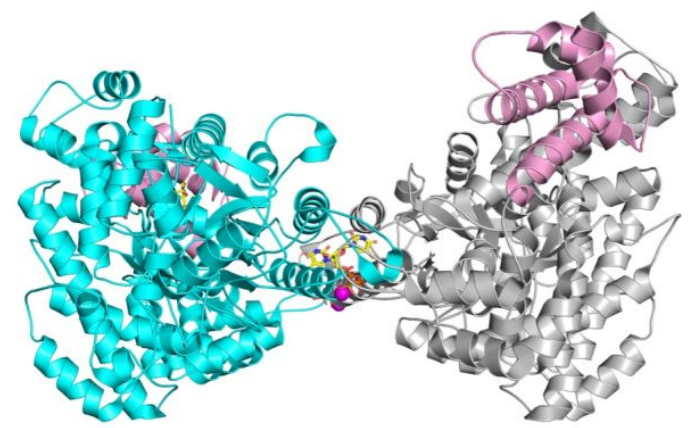

Human R1

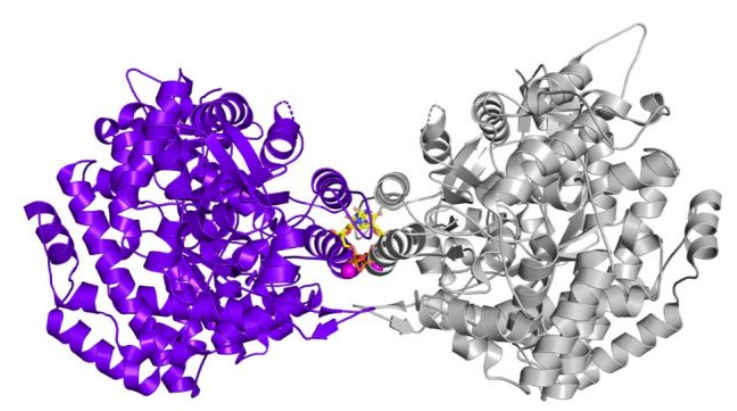

S. enterica class Ib R1

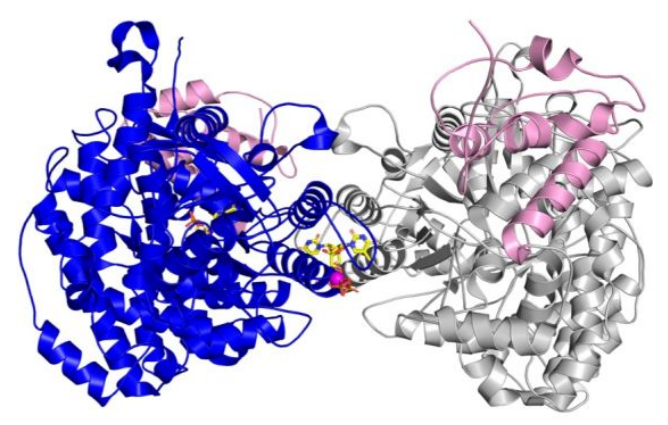

E. coli R1

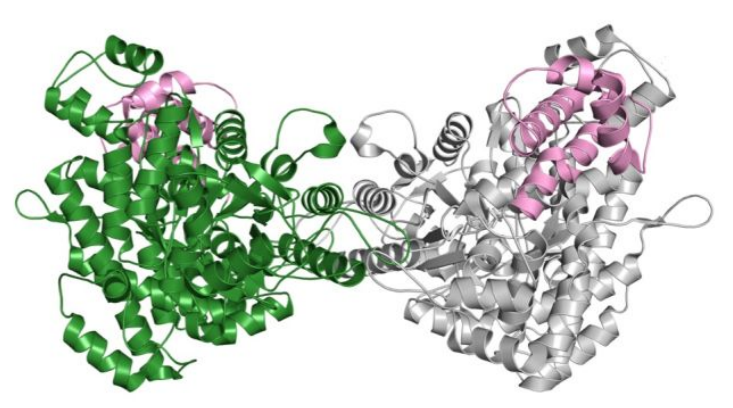

S. cerevisiae R1

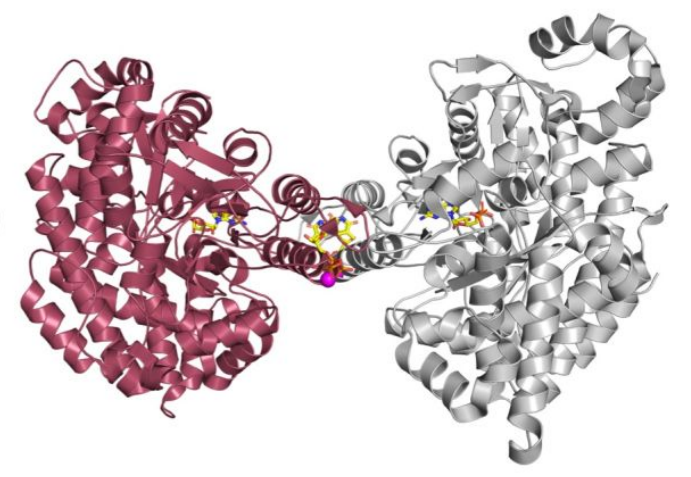

T. maritima class II RNR

Figure S1. Comparison of AaR1 with structurally similar R1 proteins. Individual monomers are depicted as ribbon representations, coloured orange and green (AaR1), blue and grey (E. coli R1, PDB ID: 6W4X), cyan and grey (human R1, PDB ID: 3HNF), green and grey (S. cerevisiae R1, PDB ID: 3PAW), purple and grey (S. enterica class Ib R1, PDB ID: 1PEQ) and burgundy and grey (T. maritima class II RNR, PDB ID: 1XJE). The ATP-cones of AaR1, E. coli $\mathrm{R} 1$, human $\mathrm{R} 1$ and $S$. cerevisiae $\mathrm{R} 1$ are shown in pink. The ATP ligands are depicted as stick models; carbon atoms yellow, oxygen atoms red, nitrogen atoms blue and phosphorous atoms orange. Magnesium ions required for ligand coordination are displayed as magenta spheres. Nucleotide ligands in each structure are shown as sticks; carbon atoms yellow, oxygen atoms red, nitrogen atoms blue and phosphorus atoms orange. A. aeolicus R1 is bound with ATP (ATPcone, a-site), E. coli R1 is bound with GDP (c-site) and dTTP (s-site), human R1 is bound with dTTP (s-site) and dATP (a-site), $S$. enterica class Ib R1 is bound with dTTP (s-site) and T. maritima class II RNR is bound with GDP (c-site) and dTTP (s-site). 

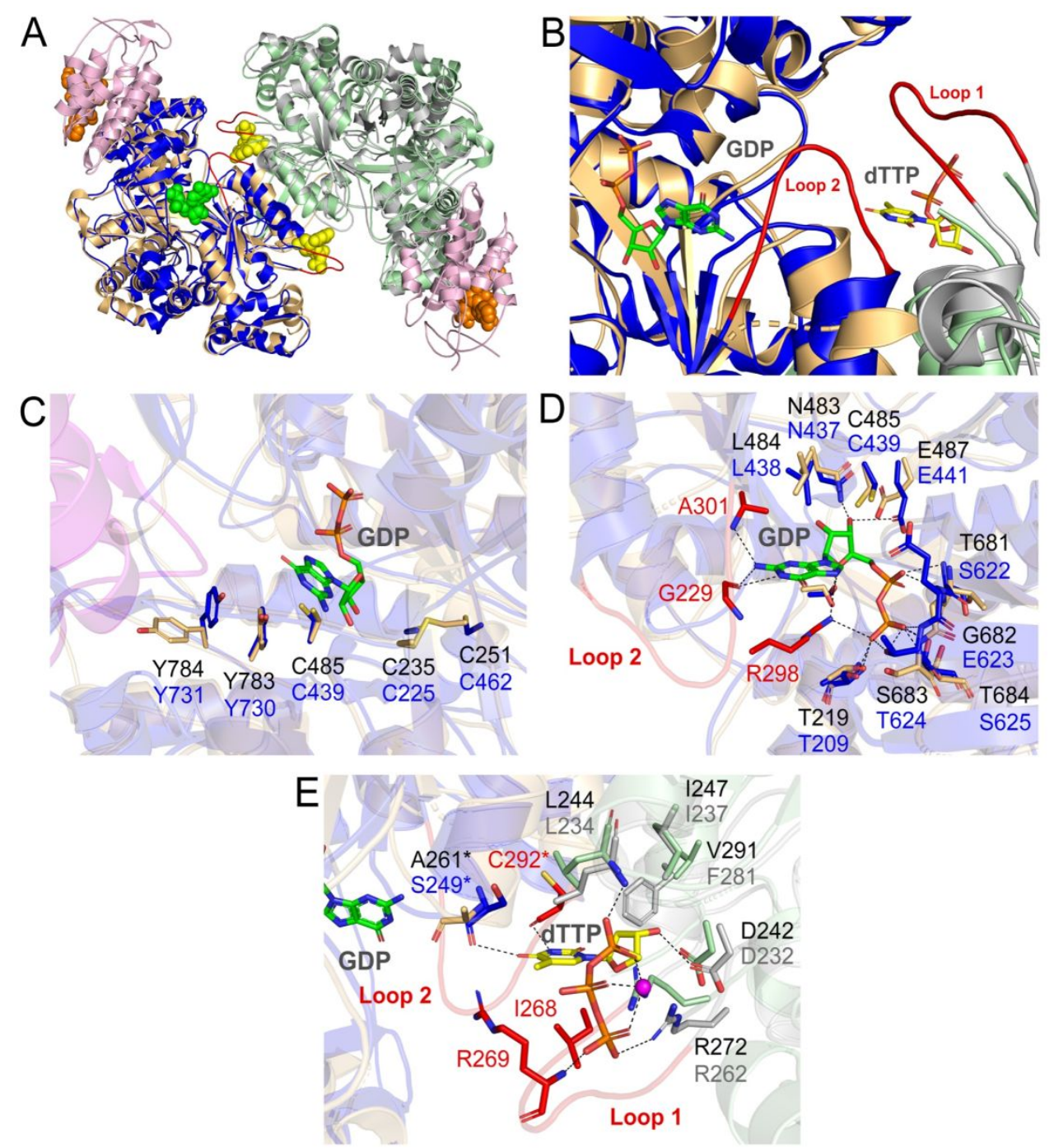

Figure S2. Comparison of AaR1 with $E$. coli R1 highlighting the c-site and s-site. (A) Superposition on AaR1 and E. coli R1, where Individual monomers are depicted as ribbon representations, coloured orange and green $(A$. aeolicus $)$ and blue and grey $(E$. coli, PDB ID: 6W4X). The ATP-cones (a-site) from AaR1 and E. coli R1 are coloured pink. ATP in the AaR1 a-site is shown in dark orange, GDP in the E. coli c-site is shown in green and dTTP in the E. coli s-site is shown in yellow. E. coli R1 loop 1 and loop 2 , which are involved in the c-and s-sites, are coloured red. These loops are disordered in the AaR1 structure. (B) Close up view of E. coli R1 loop 1 and loop 2, showing their positions relative to the c-site (GDP) and s-site (dTTP). Nucleotide ligands in this panel are shown as sticks; carbon atoms yellow (dTTP) or green (GDP), oxygen atoms red, nitrogen atoms blue and phosphorus atoms orange. (C) Comparison between AaR1 and $E$. coli R1 highlighting the two radical transferring tyrosines, the actual cysteine involved in ribonucleotide reduction (Cys485, AaR1 numbering) and two cysteines (Cys235 and Cys521, AaR1 numbering) that are oxidized upon product formation. The R2 subunit of E. coli RNR structure (PDB ID: 6W4X) is shown as a magenta cartoon. (D) Coordination of GDP in the E. coli R1 c-site compared the equivalent residues from AaR1. Hydrogen bond interactions are shown as dashed lines. (E) The hydrogen bond network of dTTP in the E. coli R1 s-site compared with the equivalent residues from AaR1. Residues from monomer 2 in both structures are labelled with an asterisk (*). 
A

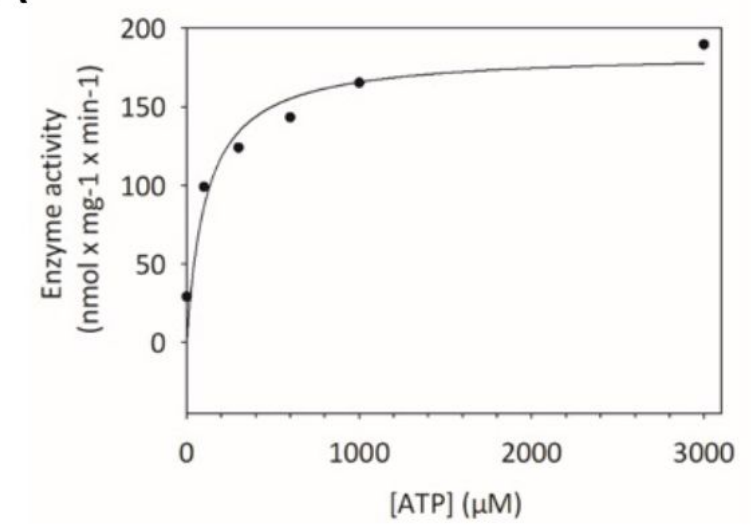

B

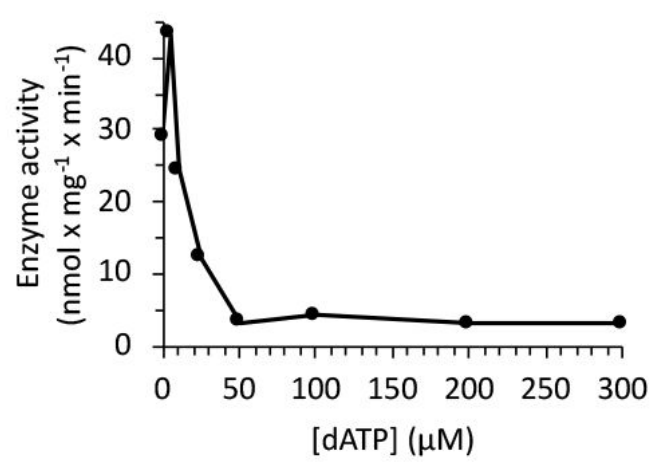

C

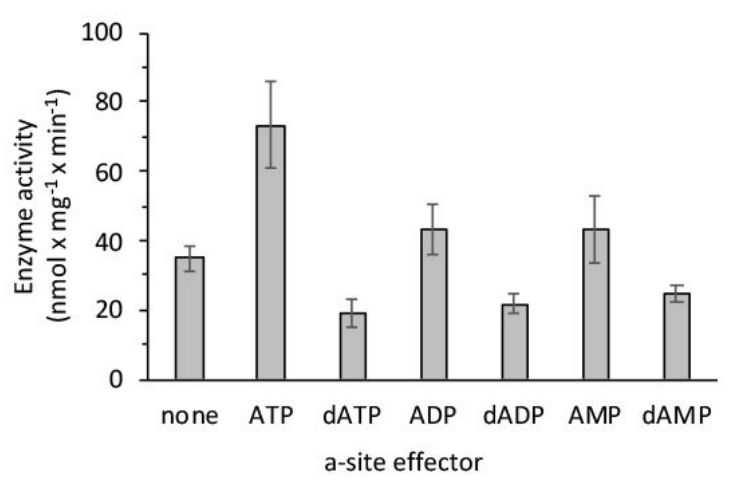

Figure S3. Activity of Aquifex aeolicus RNR in the presence of allosteric effectors. (A) ATP activates the enzyme with a $\mathrm{K}_{\mathrm{L}}$ of $113 \mu \mathrm{M}$. From a direct plot of activity versus concentration of effector, the $\mathrm{K}_{\mathrm{L}}$ value for binding of ATP to the a-site, was calculated in SigmaPlot using the equation: $V=V \max x[d N T P] /\left(K_{L}+[d N T P]\right)$. (B) dATP activated the enzyme when used at a low concentration $(5 \mu \mathrm{M})$, presumably by binding to the s-site and was inhibitory at $10 \mu \mathrm{M}$ concentration and higher. The reaction mixtures in $\mathrm{A}$ and $\mathrm{B}$ contained $4 \mu \mathrm{M}$ AaR1, $4 \mu \mathrm{M}$ AaR2 genomic, $0.5 \mathrm{mM}$ CDP substrate and the indicated amount of allosteric effector ATP or dATP. The reaction was run for 1 minute at $79^{\circ} \mathrm{C}$. (C) GDP reduction in presence of s-site saturating dTTP effector $(2 \mathrm{mM})$, and $2 \mathrm{mM}$ of the indicated adenosine nucleotides. ATP activated the enzyme, dATP, dADP and dAMP were inhibitory and ADP and AMP didn't affect the activity. Since dTTP saturated the s-site, the activation and inhibition of RNR activity occured via the a-site. Assay mixtures contained $4 \mu \mathrm{M}$ AaR1, $2 \mu \mathrm{M}$ AaR2 and $0.8 \mathrm{mM}$ GDP substrate. The reaction was run for 1 minute at $79^{\circ} \mathrm{C}$. Error bars show the standard deviation of three experiments. 


\begin{tabular}{|c|c|c|c|c|c|c|}
\hline 10 & 20 & 30 & 40 & 50 & 60 & 70 \\
\hline MEKTEKNELV & RKLIFNPQGD & REASKRKII $\bar{K}$ & GNPTNIFEL $\bar{N}$ & EIKYSWAFDI & YKLMGFTNEW' & IPEEIQMLED \\
\hline 80 & 90 & 100 & 110 & 120 & 130 & 140 \\
\hline RKQYETVLSD̄ & YEKRAYELVI & SFLIAIDSF̄̄ & VDMLKEFGRM & ITAPEVEMA $\bar{I}$ & 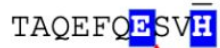 & AYSYQFILES \\
\hline 150 & 160 & 170 & 180 & 190 & & $\boldsymbol{A}^{2} \quad 210$ \\
\hline $\begin{array}{r}\text { VVDPVKADE } \bar{I} \\
\mathbf{2 2 0}\end{array}$ & $\begin{array}{r}\text { YNYWREDERL } \\
\mathbf{2 2 9}\end{array}$ & LERNKVIAEL & YNEFIRKPNE & ENFIKATIGN & YIIESLYFYS & $\underset{\boldsymbol{\Lambda}}{\mathrm{GFAFFTLGR}}$ \\
\hline QGKMRNTVQQ̄ & IKY INRDELC & FIEGTEVLTK & RGFVDFRELR & EDDLVAQYDI & ETGEISWTKP & YAYVERDYEG \\
\hline SMYRLKHPKS & NWEVVATEGH & EFIVRNLKTG & KERKEPIEKV & KLHPYSAIPV & AGRYTGEVEE & YDLWELVSGK \\
\hline GITLKTRSAV & KNKLTPIEKL & LIVLQADGTI & DSKRNGKFTG & FQQLKFFFSK & YRKINEFEKI & LNECAPYGIK \\
\hline WKKYERQDGI & AYTVYYPNDL & PIKPTKFFDE & WVRLDEITEE & WIREFVEELV & KWDGHIPKDR & NKKKVYYYST \\
\hline \multirow[t]{2}{*}{ KEKRNKDFVQ } & ALCALGGMRT & VVSRERNPKA & KNPVYRIWIY & LEDDYINTQT & MVKEEFYYKG & KVYCVSVPKG \\
\hline & 230 & $24 \underline{4}$ & $25 \underline{4}$ & 264 & $27 \underline{4}$ & $28 \underline{4}$ \\
\hline NIVVRYKDSV & CIAGNŌHVTL & FRNIINTLRK & ENPELFTPEI & EKWIVEYFKY & AVNEEIKWGQ & YVTQNQILGI \\
\hline $29 \underline{4}$ & $\omega_{30 \underline{4}}$ & $31 \underline{4}$ & $32 \underline{4}$ & $33 \underline{4}$ & $34 \underline{4}$ & $35 \underline{0}$ \\
\hline IDVLIERYIK & YLGNLRITOI & GFDPIYPEVT & NPI KWTDFF & ידסחצי & & \\
\hline
\end{tabular}

Figure S4. The nascent protein sequence of AaR2_genomic showing the position of the intein within AaR2. Residues responsible for coordinating iron are shaded blue. The tyrosyl radical residue is shaded green. The intein which is post-translationally cleaved out of the AaR2 protein is shaded grey. Two cysteine residues participating in this process are coloured red. Insertion sites self-splicing elements in other R2 sequences are indicated with arrowheads (intein, red, and group I intron, black); intein insertion sites are from several Pectobacterium phages, Corynebacterium phage Darwin, Pseudanabaena phage and Tenacibaculum phage, and group I intron insertion sites are from Bacillus amyloliquefaciens, Bacillus cereus spp, Bacillus glycinifermentans, Bacillus licheniformis, Bacillus methylotrophicus, Bacillus subtilis spp, Bacillus thuringiensis spp, Streptococcus pneumoniae spp, Thiovulum sp, Acinetobacter phage, Brochothrix phage, and Enterobacteriophage T4. 


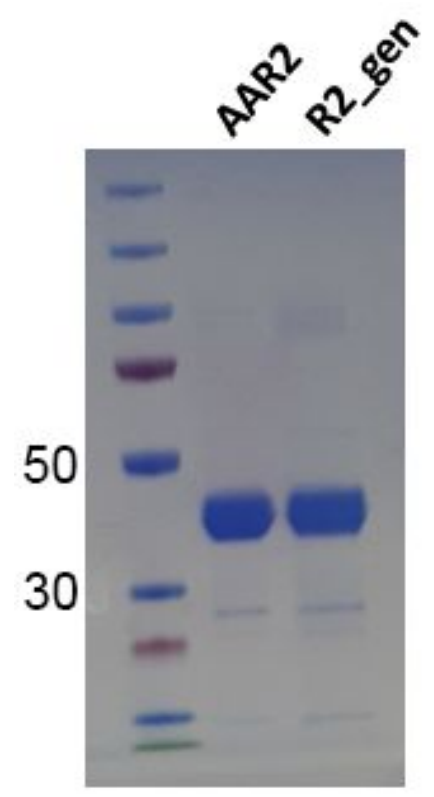

Figure S5. SDS-PAGE analysis of AaR2 and AaR2_genomic proteins following purification by IMAC and SEC. PageRuler Plus Prestained Protein ladder (Thermofischer) was used to estimate the molecular weights of the protein samples. The bands corresponding to 30 and $50 \mathrm{kDa}$ are labelled. 


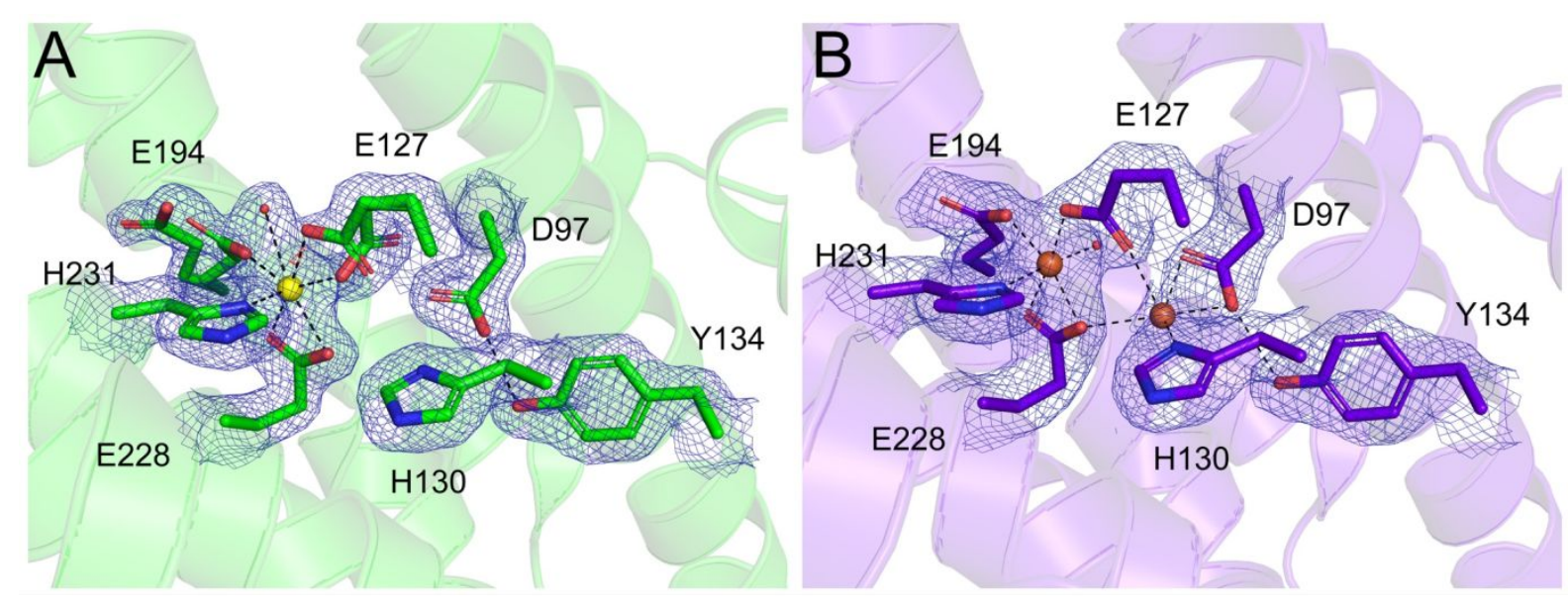

Figure S6. Iron coordination in AaR2 and mature AaR2_genomic. Hydrogen bond network of iron ions within (A) AaR2 and (B) AaR2_genomic. Iron atoms are coloured yellow (AaR2) or orange (AaR2_genomic). Amino acids contributing to metal coordination are depicted as sticks; carbon atoms green (AaR2) or purple (AaR2 genomic), oxygen atoms red and nitrogen atoms blue. The tyrosyl radical residue (Tyr134) in each structure is also shown. Hydrogen bond interactions are displayed as dashed lines. $2 F_{\mathrm{O}}-F \mathrm{c}$ electron density maps are contoured at $1.0 \sigma$ and $F \mathrm{O}-F \mathrm{c}$ electron density maps are contoured at $3.0 \sigma$. Relevant water molecules in each structure are shown as red spheres. 


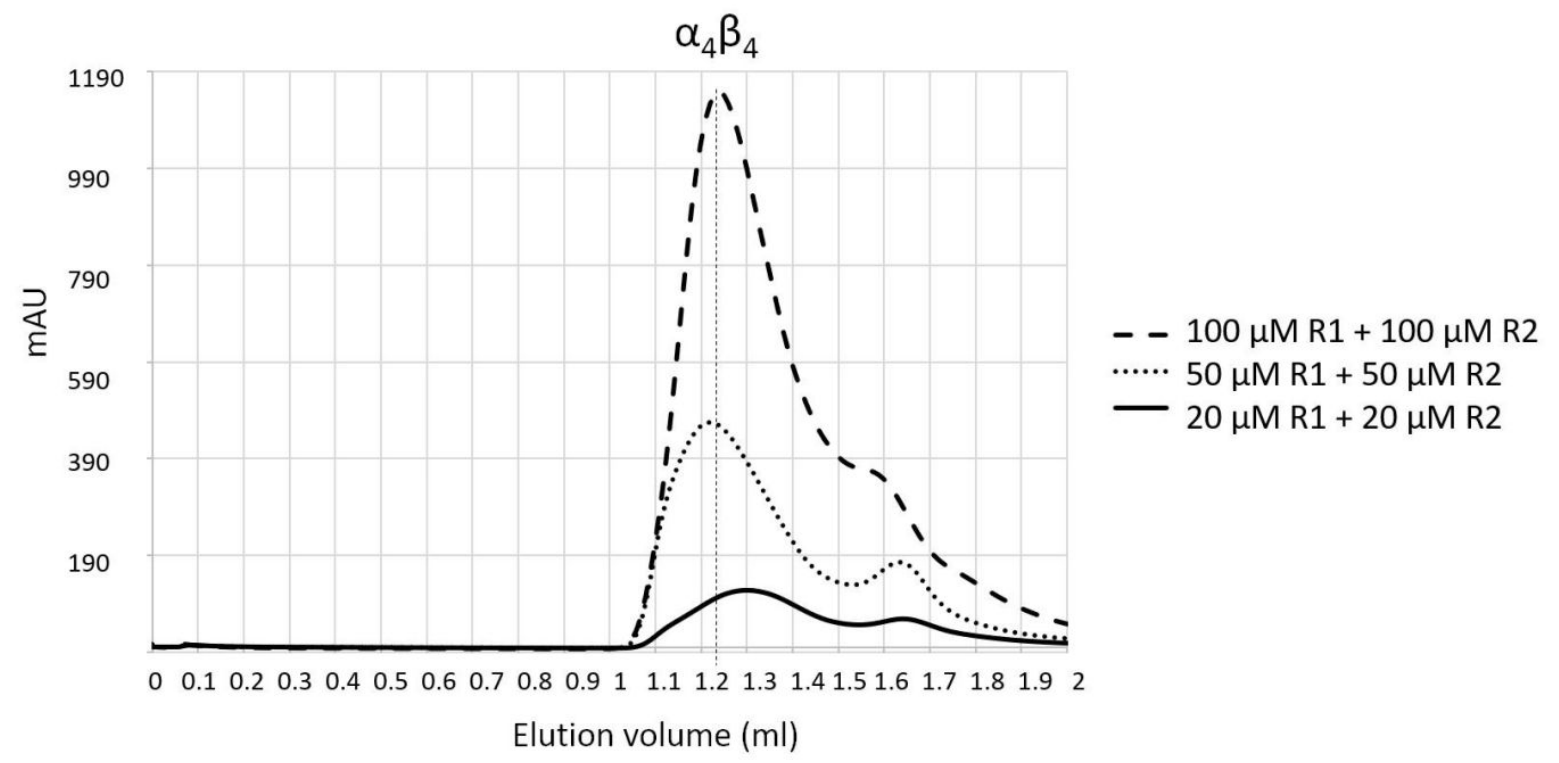

Figure S7. Size exclusion chromatography analysis of AaR1 and AaR2 using different protein concentrations. Experiments were performed in the presence of 5 mM ATP with either $100 \mu \mathrm{M}$ AaR1 $+100 \mu \mathrm{M}$ AaR2 (dashed line), $50 \mu \mathrm{M}$ AaR1 +50 $\mu \mathrm{M}$ AaR2 (dotted line) or $20 \mu \mathrm{M}$ AaR1 $+20 \mu \mathrm{M}$ AaR2 (solid line). 